Relations industrielles

Industrial Relations

\title{
Fragments sur l'essentiel du droit de l'emploi, par Fernand Morin, Montréal : Wilson et Lafleur, 2007, 300 p., ISBN 978-2-89127-790-7.
}

\section{Mélanie Gagnon}

Volume 63, numéro 2, 2008

URI : https://id.erudit.org/iderudit/018587ar

DOI : https://doi.org/10.7202/018587ar

Aller au sommaire du numéro

Éditeur(s)

Département des relations industrielles de l'Université Laval

ISSN

0034-379X (imprimé)

1703-8138 (numérique)

Découvrir la revue

Citer ce compte rendu

Gagnon, M. (2008). Compte rendu de [Fragments sur l'essentiel du droit de l'emploi, par Fernand Morin, Montréal : Wilson et Lafleur, 2007, 300 p., ISBN 978-2-89127-790-7.] Relations industrielles / Industrial Relations, 63(2), 378-380. https://doi.org/10.7202/018587ar

Tous droits réservés (C Département des relations industrielles de l'Université Laval, 2008
Ce document est protégé par la loi sur le droit d'auteur. L'utilisation des services d'Érudit (y compris la reproduction) est assujettie à sa politique d'utilisation que vous pouvez consulter en ligne.

https://apropos.erudit.org/fr/usagers/politique-dutilisation/ 
was concerned about the author's rather dogmatic style of writing. For example, he defines globalization as "the gradual reduction in the costs of trade" (p. 6). I am aware of other definitions and would have appreciated preamble about the fact that there are various points of view and that, for present purposes, a particular one is chosen.

Second, limited discussion is given of how the book's theory was developed. For example, was the "star analysis" concept created based on application of the author's logic and polemic, did practicing managers suggest its elements in light of personal experience, or was another method used? How should we weight the importance of the model's various features? I raise these matters to indicate my disquiet that scholarship about corporate strategy does not pay sufficient heed to conventional benchmarks for quality research. Another example concerns the generic strategies that firms may adopt. In the book, I found insufficient detail about the process the author undertook to identify and/ or validate these. Certainly, ideas seem to make sense and it does appear from the case studies that they have real-world utility. However, such impressions may be deceiving. Could we-as readers - be being duped by only having examples presented to us that support the principle (i.e. data-mining)? The answer to this question could be provided through use of sampling and statistical inference. If such analyses were beyond the scope of the project or would have disrupted the flow of the argument, their relevance could still have been discussed.

Third, theory about strategy development may be overly generic. When I read Spulber's range of options, I contemplated how a commercial entity may pursue alternatives. This raises the question: what evidence is there that a given set of strategy options accounts for all possible approaches that a firm may pursue? Some commentary on this issue would have given the book more substance. My bias would have been to either provide such additional information or, at least, flag the issue.

Overall, Spulber's book provides a solid contribution to its field. The work introduces a substantial amount of new and innovative material. It does not necessarily create genuinely original theoretical paradigms but certainly pushes the conventional ones to their limits. For this reason alone, it is at least as good as other well known books about commercial strategy. I have suggested to my master's degree students that they should read Spulber's latest work. I have told them that I think it contains ideas which are novel and intuitively appealing. However, I have invited those with a research bent to consider how material may be further tested and evaluated. When such further scrutiny occurs, we will know whether Spulber has written something that is merely practical, helpful and well written or a genuine classic.

Anthony M. Gould Laval University

\section{Fragments sur l'essentiel du droit de l'emploi,}

par Fernand MoRIN, Montréal : Wilson et Lafleur, 2007, 300 p., ISBN 978-2-89127-790-7.

L'objectif poursuivi par l'auteur est simple. Il partage ses notes et observations à l'égard de différentes facettes du droit de l'emploi en mettant l'accent sur les questions relatives aux justificatifs et aux sources des règles de droit (omettant sciemment toute transposition technique sous-jacente), interrogations qui expliquent sa nécessaire raison d'être au plan social, économique et juridique. C'est par le truchement de vingt-cinq billets portant sur autant de facettes 
du droit de l'emploi que l'ouvrage est présenté. Ne pouvant, aux fins du présent exercice, fournir la nomenclature complète des thématiques abordées nous nous attardons ici à la description et à l'appréciation du volume en ce qu'il a d'essentiel.

La première partie énonce les principes généraux du droit de l'emploi. Sur ce point, l'auteur prend soin d'expliciter les raisons politico-historiques ayant conduit l'État à légiférer en cette matière. Le droit de l'emploi demeure essentiellement voué à la protection de la personne du salarié, c'est pourquoi il est constitué de façon à rééquilibrer le rapport inégalitaire entre les parties.

La deuxième partie, composée de dix « fragments », se penche sur la relation de travail et sur le contrat de travail. L'auteur argue qu'il serait imprudent de rejeter le contrat de travail sans lui avoir trouvé, au préalable, un substitut, le contrat étant à même de «saisir suffisamment les données principales de la relation de travail et [de] les aménager en un tout assez cohérent et pratique ». Cette prise de position à l'égard de l'importance du Code civil du Québec comme source juridique est en filigrane tout au long de l'ouvrage. En effet, l'auteur fonde une partie importante de son analyse sur le Code civil, notamment eu égard à sa disposition préliminaire et à l'article 2085 , où se trouve la définition du contrat de travail. En conséquence, il expliquera que les dispositions prévues à la Loi sur les normes du travail au sujet de l'obligation de disponibilité constituent des limites à cette obligation. Il est dès alors loisible au salarié de se déclarer indisponible en ces périodes, et ce, sans que l'on puisse y associer un quelconque manquement à ses obligations contractuelles. La notion de dignité du salarié constitue un autre sujet abordé dans cette partie. Ainsi, l'auteur soutient que le fait d'accepter de travailler "sous la direction et le contrôle de l'employeur» (art. 2085 C.c.Q.) ne peut servir de sauf-conduit pour réduire ou occulter le droit à la dignité, compte tenu surtout de l'obligation faite à l'employeur à l'article 2087 C.c.Q. de veiller à assurer le respect de la dignité du salarié. Enfin, le dernier « fragment» traite de la mise à pied et de la situation juridique du salarié pendant cette période de « limbes ». Après avoir passé en revue les règles de droit applicables (L.N.T.), dans lesquelles il perçoit un manque de limpidité, l' auteur se désole de l'absence d'arrimage entre ces dernières et les dispositions du Code civil.

Dans la troisième partie du volume, l'auteur jette d'abord un regard sur les effets du droit de l'emploi sur l'employeur. Il appert que ce corpus législatif constitué des lois du travail est favorable aux employeurs, parce que la concurrence entre ceux-ci s'effectue sur une base égalitaire et aussi parce qu'en leur absence ils connaîtraient davantage de tumulte et de désordre. Ensuite, l'auteur aborde aux fragments « $\mathrm{N}$ à $\mathrm{Q}$ », les thématiques liées au pouvoir de discipline de l'employeur. La justification de la notion de privilège du préalable qui échoit à l'employeur y est développée de façon limpide comme étant un produit dérivé du droit de propriété. L'employeur pouvant exercer son pouvoir sur son personnel, il est dès lors logique qu'il lui revienne de justifier ses actions après coup. Quant au fondement du pouvoir disciplinaire de l'employeur, l'auteur mentionne à juste titre qu'aucune loi n'en traite directement. Ce faisant, c'est avec peu d'étonnement que le lecteur prendra connaissance de la posture de l'auteur à ce sujet : l'employeur dispose de la légitimité d'imposer des sanctions lorsqu'il y a manquement professionnel, puisque le salarié s'oblige, en vertu de son contrat, à travailler sous le contrôle de l'employeur.

La quatrième et dernière partie de l'ouvrage est consacrée à l'étude des rapports collectifs du travail de même qu'au caractère singulier de la conven- 
tion collective. Y est exposé succinctement, mais efficacement, le «refus implicite » du législateur de permettre à tous les travailleurs d'exercer pleinement leur droit d'association. L'auteur étaye sa posture théorique en expliquant qu'un fort pourcentage de travailleurs ne correspond plus à la définition de salarié contenue dans le Code du travail, les types d'emplois s'étant multipliés au cours des dernières années. En corollaire, considérant l'entendement jurisprudentiel de la définition de salarié, un nombre élevé de travailleurs est écarté de l'accès à un régime de rapports collectifs.

L'auteur, porte-étendard de la thèse de la coexistence de ces deux actes juridiques que sont le contrat individuel et la convention collective, est fidèle à cette dialectique tout au long de ce chapitre. Le lecteur averti ne sera guère surpris de cette prise de position, cette dernière ayant été maintenue et étayée au fil des ans. Retraçant la chronologie des différents jugements de la Cour suprême au sein desquels des définitions de la convention collective sont fournies d'abord celle du tripartisme et ensuite celle de la suspension du contrat de travail -, l'auteur soutient que le Code civil n'évacue pas de son application le salarié qui se trouve sous «l'écran syndical ». À son avis, une conception contraire ne serait pas conforme à l'esprit du droit, à savoir le respect de la personne salariée. De fait, la finalité des rapports collectifs n'est pas de substituer la convention au contrat individuel, mais de donner à celui-ci un contenu réellement négocié. Cette thèse est cependant diamétralement opposée à celle des tenants de l'école orthodoxe pour qui le contrat civil n'a plus sa place en contexte de rapports collectifs du travail.
Compte tenu de l'angle d' analyse retenu par l'auteur, celui-ci ne pourra rallier l'ensemble des juristes et des praticiens, son ouvrage constituant un plaidoyer en faveur de la thèse de la coexistence de la convention collective et du contrat de travail.

L'ouvrage possède le défaut de ses qualités à savoir que ces «fragments », pour reprendre le vocable de l'auteur, ont été revisités, ayant déjà fait l'objet de publications antérieures. Conséquemment, le reproche que l'on peut faire est qu'il comporte certaines répétitions susceptibles de lasser le lecteur : notamment, la référence à l'article 2085 du Code civil de même que celle en regard de l'importance de sa disposition préliminaire. Nonobstant cet élément lacunaire, s'il en est un, le volume constitue un incontournable pour la discipline. Les autres thématiques couvertes par l'auteur, même si elles n'ont pas été traitées dans la présente recension, méritent qu'on s'y attarde. Tel est le cas, par exemple, des billets traitant de la liberté des parties à la convention et de l'ordre hiérarchique des règles de droit, de l'obligation de dénonciation, de la validité des clauses de dernière chance et du privilège de souscription d'actions des gestionnaires.

En guise de conclusion, cette recension serait incomplète si nous omettions de souligner que nous sommes en présence d'un ouvrage de grande qualité où l'argumentaire est exposé de façon habile et limpide, tout cela bien entendu en restant fidèle à ce que l'on connaît de l'auteur, c'est-à-dire un style littéraire riche et coloré qui ne peut que captiver le lecteur.

Mélanie Gagnon

Université du Québec à Rimouski 